\title{
Complex Structures: A Symbiosis of Experiments and Numerical Studies
}

\author{
J. H. Bilgram and H. M. Singer
}

\begin{abstract}
Dendrites are the best studied structures formed during solidification of undercooled melt. Additionally other complex structures have been found in experiments and numerical studies. We perform 2D/3D-numerical simulations of solidification processes and experiments which allow in situ investigations of three-dimensional growth of xenon crystals into undercooled pure melt. Dendrites, seaweed, doublons, triplons etc. can be produced in experiments and numerical studies depending on initial conditions. In an interplay of numerical studies and experiments we use simulations to interpret and to plan experiments. Experimental results are used as a basis of model calculations and the development of models of complex shapes. Three-dimensional growth shapes of crystals are reconstructed using sophisticated image processing combined with experimentally determined shape parameters.
\end{abstract}

\section{1 \\ Introduction}

Applications in automotive industry call for aluminium castings to be mass-produced without casting defects. Predictive models are necessary to prevent such defects. During the growth of a solid from its undercooled parent melt microstructures are formed. The best-known cases of such structures are dendrites. Microstructural changes can be observed as undercooling is increased, namely the typical length scales like the tip radius decrease with increasing undercooling [1, 2]. These are quantitative changes. In addition to that, for some materials it has been observed that they also undergo an abrupt decrease in microscale with increasing undercooling. This effect is known as spontaneous grain refinement. It has been observed in solidification from undercooled pure metallic melts [3] and alloys [4, 5]. Evidence has been found that spontaneous grain refinement is initiated by changes in the morphology of the microscopic structures [3]. This is in difference to the above mentioned quantitative grain refinement with increasing undercooling. In order to obtain more information on shapes, developed during solidification, numerical modeling and analytical stu-

Solidification and Crystallization. Edited by Dieter M. Herlach Copyright (C) 2004 WILEY-VCH Verlag GmbH \& Co. KGaA, Weinheim ISBN: 3-527-31011-8 
dies of the solidification process have been performed. Starting with a mathematical formulation of the dendrite problem, the goal is to find out which solutions exist and which morphologies are possible. Namely the stability of solutions has been studied $[6,7,8]$. A very astonishing result was the discovery of a doublon in $2 \mathrm{D}$ studies [9]. It is formed by two crystalline tips with a straight channel in between. This is a typical non-equilibrium shape. It was followed up by numerical studies which found a triplet in an 3D channel [8]. In a next step a morphology diagram has been developed for 2D systems $[10,11]$. Regions for stable fractal and compact growth of dendrites and seaweed have been determined. Seaweed has been considered to be built up from doublons. In this study the stability regions of morphologies have been determined in a field of supercooling of the melt vs. anisotropy of the surface free energy. Supercooling is given in the range from 0 to 1 in dimensionless units and the anisotropy in the range from 0 to 1 . High anisotropies lead to dendritic growth, at low anisotropy and sufficient undercooling seaweed is growing. The amount of noise in the system under consideration may influence the position of the phase boundaries. Therefore the phase diagram has to be considered to be a qualitative one.

To verify predictions, numerical and experimental studies have to be combined. We hope that such combined studies will stimulate the application of theoretical results in the design casting processes.

\section{2}

\section{Experimental Studies}

Existing models for dendritic growth do not predict morphological parameters with sufficient accuracy for reliable calculations to be applied in industrial casting processes. Metallographic techniques show microstructures which have been transformed during solidification and subsequent cooling. The study of decanted structures leads to an overestimation of the fraction of solid and does not provide information of solidification kinetics. Time resolved synchrotron radiation imaging techniques do no not provide a lateral resolution suficient to determine typical length scales of the fine structure and it is not possible to study three-dimensional structures with this technique up to now [12]. Thus the use of transparent materials is the only way to determine growth parameters of morphologies during the solidification process. Two types of experiments have been performed: quasi two dimensional studies on organic alloys [13] and studies of pure materials during free growth in three dimensions $[14,15,16]$.

First experimental evidence of doublons has been achieved by Akamatsu et al. [13]. A thin cuvette was used and the material was an mixture of two organic liquids $\mathrm{CBr}_{4}$ and $\mathrm{C}_{2} \mathrm{Cl}_{6}$. This system has two properties, which are important for the experiment: i) An alloy is used. It is well known that in alloys much higher constitutional undercoolings are possible than for bulk undercoolings in a pure melt.

ii) Interface kinetics and surface free energy of the solid-liquid interface are anisotropic. Therefore this system has the great advantage that it is possible to change anisotropy of the surface free energy by changing the orientation of the seed crystal. 
In these experiments the growth of seaweed has been observed and phase diagrams of the type as developed by Brener et al. [11] have been verified.

The calculations of Brener et al. have been performed for a 2D system. The experiments of Akamatsu et al. have been performed in a thin cuvette and thus it is a quasi-two-dimensional system. A two-dimensional doublon is topologically different from a doublon in three dimensions, because in 2D there is only one way out of the channel, namely at the tips. In the 3D case it is possible to move out of the channel in a direction perpendicular to the plane defined by the two branches limiting the channel. This fact changes topology significantly and also the possibility for the latent heat to diffuse away.

Experiments in three dimensions to detect doublons and seaweed have been performed in a growth vessel with a volume of $100 \mathrm{~cm}^{3}$ of pure liquid xenon. We use xenon as a transparent model substance for metals, because it forms a simple liquid, it crystallizes in an fcc-lattice, and it forms a solid-liquid interface which is rough at atomic scale. Details of the experimental setup are described in [16, 17]. At the beginning of the experiment the melt is brought to a homogeneous temperature $T_{\infty}$ which is below the melting temperature, i.e. the melt is homogeneously undercooled. Then a crystal is nucleated in a capillary and grows through the capillary into the undercooled melt. The dimensions of the growth vessel are sufficiently large to ensure free growth of the crystal. According to the properties of the solid-liquid interface and the undercooling in the range of $10^{-3}$ to $10^{-2}$ in dimensionless units, a dendrite grows to a stationary state after several minutes and the temperature distribution around the dendrite reaches a steady state. An important feature of the experimental setup [17] is the possibility to shift this dendrite up and down inside of the growth vessel. Shifting the dendrite in the growth vessel means to disturb the temperature distribution around the crystal and to wash off the isotherms. Immediately after such a shift the crystal is surrounded by melt with a spatial homogeneous temperature. Any surface element of the dendrite tip is in contact with liquid at a temperature close to $T_{\infty}$. The thermal gradient at the crystal surface is everywhere the same. (Neglecting that the surface has been non isothermal before shifting the crystal.) Therefore any surface element of the crystal is growing with the same growth rate. This leads to an increase in the tip radius of the dendrite (Figure 1.) With increasing tip radius the dendrite reaches a state where the tip becomes unstable and tip splitting is observed. After tip splitting two tips continue to grow and form a doublon with a straight channel between the two tips. A sequence of contours is shown in Figure 1. By this procedure it is possible to grow various morphologies. Figure 2 shows a dendrite, a doublon and a seaweed morphology.

In difference to Brener et al. [11] we distinguish between doublon and seaweed morphology. This difference may originate from the three-dimensional geometry. The three morphologies can be distinguished from symmetry considerations: A dendrite shows a high degree of symmetry. For the seaweed no growth direction can distinguished in the plane of projection. The doublon has an axis of symmetry in the center of the channel.

In three dimensions the morphological transitions are not limited to the three cases shown in Figure 2. In addition to that we find triplons and quadruplons and 


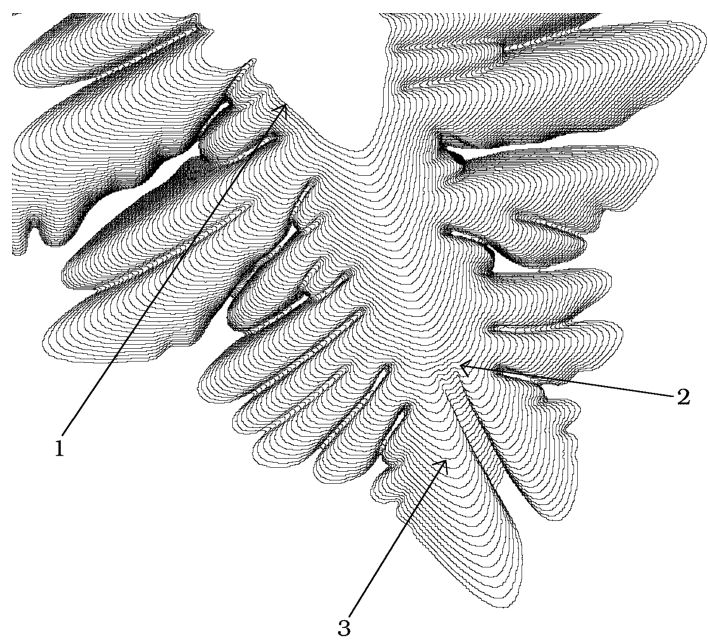

Fig. 1. A sequence of contours taken after the crystal has been shifted in the growth vessel. First we have the shape of the dendrite (arrow 1). During the temporal development the tip radius increases and the tip radius becomes unstable (arrow 2 ). Finally two tips are formed with a straight channel of liquid in between forming a doublon (arrow 3).
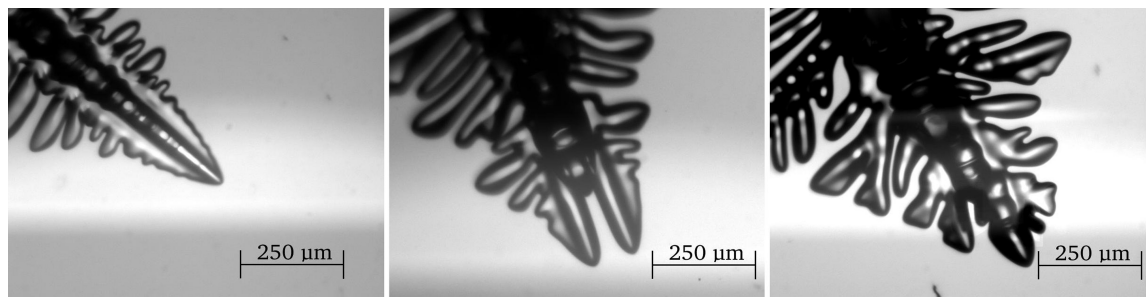

Fig. 2. Three morphologies: A dendrite, a doublon, and a seaweed.

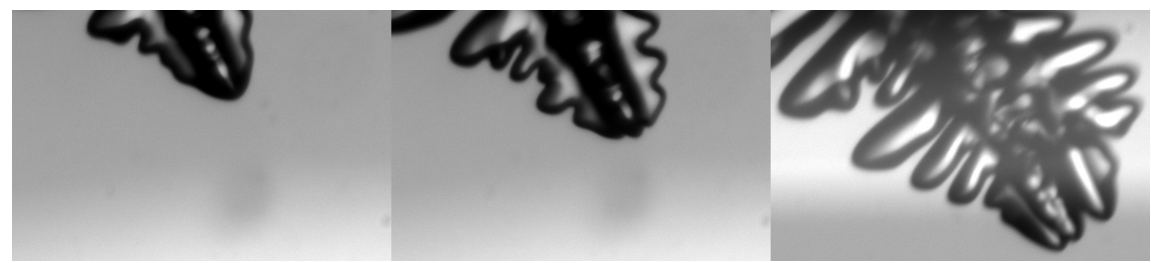

Fig. 3. A crystal with three tips is formed in a similar process as observed in Figure 1.

even higher multiples of tips. Up to now we do not know the precise conditions for the occurrence of a special morphology. Figure 3 shows the development of a triplon out of a dendrite. 


\section{Numerical Studies}

In order to verify and refine the qualitative analytical predictions by Brener et al. [11] we have performed phase field simulations with two different phase field models in 2D and 3D. Phase field models are an elegant approach to solve the analytical equations numerically. Instead of calculating a sharp phase boundary between solid and liquid in the Stefan- or sharp interface-problem every grid point holds in addition to the temperature also its phase state. The phase may have arbitrary values between 0 (solid) and 1 (liquid). A thin, but finite region of steep changes between solid and liquid models the interface. This width can be chosen and determines how well the approximation is in accordance with the sharp interface equations.

For our investigations we have chosen the models of Wheeler et al. [18] and Karma et al. [19]. The main difference between the two models is that in the Wheeler model the ratio between kinetic and capillary terms is fixed, where as in the Karma model the kinetic term can be cancelled by appropriately chosen terms for low undercoolings. We have performed simulations on a $2000 \times 2000$ grid with different undercoolings and anisotropies. The anisotropy axis goes from 0 to $6.5 \%$ as this is the maximal value where a crystal still grows in rough growth. For higher anisotropies the crystal becomes facetted as there occur forbidden growth directions. An initial coarse scan of 100 simulations was used to partition the plane. Subsequently a binary search for the morphology boundary was performed. By this refinement we were able to tie up the uncertainty region for the morphology boundary to up to $10^{-2} \%$. For lower undercoolings the simulation time increases significantly, therefore the uncertainty becomes bigger. In Figure 4 the results for the 2D simulations can be seen for both models. We have identified 3 regions of different morphologies: for low anisotropies seaweed structures (sw) and for high anisotropies dendrites are observed (den). For intermediate ansiotropies and high enough undercoolings doublons are found $(\mathrm{db})$. Both models show a similar behavior as the analytical predictions for the morphology boundary (seaweed-dendrite): The shape is concave for increasing anisotropy. However there is a big difference to the predictions for low undercoolings: while the predictions state that the whole shape of the boundary is concave we find in our simulations for low undercoolings and anisotropies a convex boundary. Comparing the two phase field models with each other we find qualitative correspondence. However we state that the boundary of the Karma model is slightly more on the left than the one of the Wheeler model. A detailed discussion of this behavior can be found in [20].

Three-dimensional simulations are very time-consuming. Even though we use an adaptive mesh code which runs parallel on 32 processors, it was not possible to obtain the same fine resolutions as for 2D simulations. As we have found qualitative agreement between the two models we have preformed 3D simulations in a domain $400^{3}$ only for the Karma model. The results are qualitatively similar to the 2D case however with two exceptions: i) The morphology boundary for the same parameters as in the $2 \mathrm{D}$ case is shifted along the anisotropy axis to the right by an amount of $0.32 \%$. This result can be explained by the topological difference between $2 \mathrm{D}$ and 


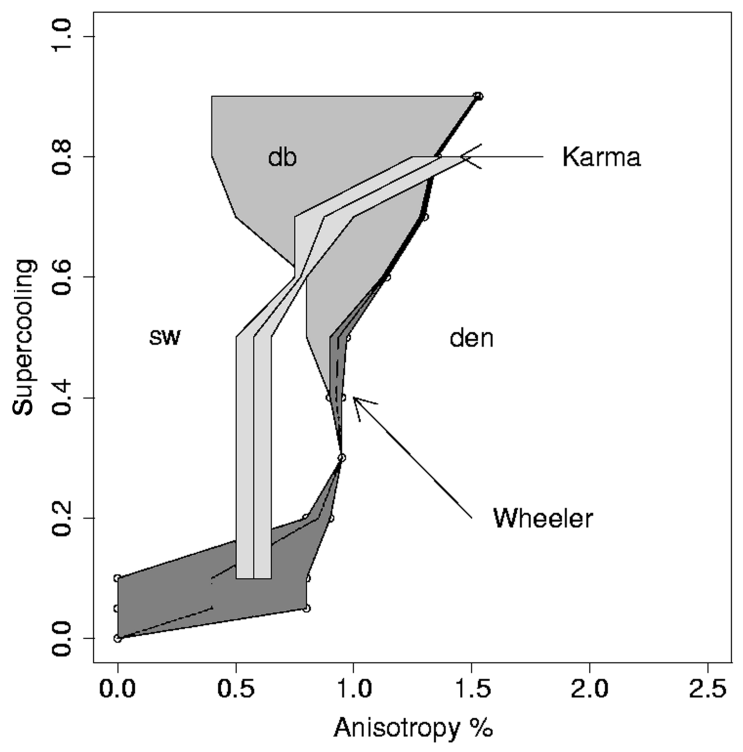

Fig. 4. Morphology diagram in 2 dimensions of the Wheeler model and the Karma model [20]. The zones marked with Wheeler and Karma correspond to the uncertainty regimes given by these models. The dendritic domain is denoted as "den". Seaweed as "sw" and doublon as "db".

3D: in 3D there is one additional dimension where heat can be transported away, therefore more anisotropy is needed to stabilize the structure to be dendritic. ii) No doublon region at all was found in $3 \mathrm{D}$ as opposed to $2 \mathrm{D}$ where we also started all simulations with the same initial conditions (a small spherical seed) and found a distinct doublon region. When starting with a spherical seed in 3D only seaweeds or dendrites can be simulated. It was however possible to simulate 3D doublons nevertheless by using special initial conditions [20]: by placing two identical seeds at a large enough distance, both start growing as a dendrite. However very soon they start interacting with each other and instead of developing four fins each they grow in parallel and develop only 3 fins each as observed in our experiments (Figure 3).

\section{4}

\section{Conclusions}

Quenching of metals during solidification provides some information on microstructures but not on the dynamics of morphology transitions during the solidification process. Transparent substances have been used to simulate solidification of metals. Rare gases have low melting entropy and form a "simple liquid“ similar to metals and can therefore be used as model substances to simulate transparent metals. In these systems the solidification process can be studied in situ. Up to now the in- 
formation from model experiments with transparent model substances has been limited because no 3D information on the microstructure formed during solidification was available.

Two new features have been introduced into the study of solidification by model experiments: 1.) the study of various morphologies and morphological transitions, and 2.) the possibility to reconstruct the shapes of transparent 3D objects [21]. Morphology changes seem to lead to an explanation of grain refinement in metals, a feature that remained unexplained since a long time. The determination of the $3 \mathrm{D}$ shape of objects formed during freezing provides for the first time the possibility to determine 3D shape parameters necessary to apply theories in industrial processes. We have started to determine such parameters and to characterize various morphologies formed during solidification and their transitions. We have the unique possibility to combine theoretical studies with experiments.

The interaction of experiment and theory is crucial. Theoretical studies are stimulated by unexpected experimental observations, and using an expression coined by L. Pasteur, the eye of the experimentalist is trained by theoretical results to see new structures.

\section{Acknowledgements}

We thank Professor Dr. H. R. Ott for his support of our experiments. This work was supported by the Swiss National Science Foundation.

\section{References}

[1] J.P. Gollub and J.S. Langer, Rev. Mod. Phys. 1999, 71, S396-S403

[2] A. Karma in Banching in Nature (Ed.: V. Fleury, J.-F. Gouyet, and M. LeoN ETTI) Springer, Berlin, 2001, Chapter 9

[3] K. Dragevski, R. F. Cochrane, and A.M. Mullis, Phys. Rev. Lett. 2002, 89, 215502

[4] R. Willnecker, D.M. Herlach, and B. Feuerbach, Appl. Phys. Lett. 1990, $56,324-326$

[5] M. Schwarz, A. Karma, K. Eckler, and D.M. Herlach, 1994, 73, 1380-83

[6] M. Ben Amar and E. Brener, Phys, Rev. Lett. 1995, 75, 561-564

[7] T. Ihle, and H. Müller-KrumbhaAR, Phys. Rev. Lett. 1993, 70, 3083-86

[8] T. Abel, E. Brener, and H. MüllerKrumbhaar, Phys. Rev. E, 1997, 55, 7789-92
[9] T. IHLE, Wachstumsmuster unter diffusivem Transport, Berichte des Forschungszentrums Jülich, Jülich, 1996, Vol.3230,

[10] E. Brener, H. Müller-KrumbhaAR, and D. Temkin, Phys. Rev. E, 1996, 54, 2714-22

[11] E. Brener, H. Müller-KrumbhaAr, D. Temkin, and T. Abel, Physica A $1998,249,73-81$

[12] R.H. Mathiessen, L. Arn berg, K. Ramsoskar, T. WeitKamp, C. Rau, and A. SNigirev, Metall. Mater. Trans. B, 2002, 33B, 613-623

[13] S. Akamatsu, G. Faivre, and T. Ihle, Phys. Rev. E 1995, 51, 4751-73

[14] M.B. Koss, J.C. LaCombe, L.A. TennenHOUSE, M.E. GLicKSMAN, and E.A. Winsa, Metall. Mater. Trans. A, 1999, 30A, 3177-90. 
[15] D. P. Corrigan, K. B. Koss, J.C. LACombe, K.D. De Jager, L.A. TennenHouse, and M.E. Glicksman, Phys, Rev. E, 1999, 60, 7217-7223

[16] U. BisAng and J.H. Bilgram, Phys. Rev. E, 1996, 54, 5309-26

[17] I. Stalder and J.H. Bilgram, Europhys. Lett. 2001, 56, 829-835
[18] A.A Wheeler, B.T. Murray and R.J. Schaffer, Physica D, 1993, 66 , 243-262

[19] A. Karma and W.-J. Rappel, Phys. Rev. E 1998, $57,4323-49$

[20] H.M. Singer, I. Loginova, J.H. BiLGRAM, and G. AmberG, J. Cryst. Growth, submitted

[21] H.M. Singer and J.H. Bilgram, this volume 